\title{
Pregnancy with third degree uterine prolapse: a rare case report
}

\section{Latika*, Smiti Nanda, Meenakshi Chauhan, Vani Malhotra}

Department of Obstetrics \& Gynaecology, PT. B. D. Sharma, PGIMS, Rohtak, Haryana, India

Received: 06 December 2015

Accepted: 07 January 2016

\section{*Correspondence:}

Dr. Latika,

E-mail: latika.duhan@gmail.com

Copyright: (C) the author(s), publisher and licensee Medip Academy. This is an open-access article distributed under the terms of the Creative Commons Attribution Non-Commercial License, which permits unrestricted non-commercial use, distribution, and reproduction in any medium, provided the original work is properly cited.

\begin{abstract}
Occurrence of prolapse of uterus during pregnancy is an extremely rare event. Less than 300 cases have been reported in the literature till date. Incidence of uterine prolapse in pregnancy is 1 in 10,000-15,000 deliveries worldwide. A 35 year old G5P3L1A1 with history of five months amenorrhea was referred to our hospital in view of uterine prolapse. On enquiring she gave history of something coming out of vagina since one month. On local examination, third degree uterovaginal prolapse with cord prolapse seen. Patient went into labour spontaneously and expelled an abortus. She was discharged next day with advice regarding follow up visit after six weeks. Uterine prolapse during pregnancy is a rare occurrence which increases both maternal and fetal morbidity. There are many etiological factors which have been cited to be responsible for this condition, important ones are: Multiparity, vaginal delivery, advanced maternal age, increased body mass index. Elective caesarean section can be planned in cases with edematous and elongated cervix. Individualized approach and careful monitoring is the key to manage such patients. Uterine prolapse during pregnancy is a rare event. While managing such patients, an obstetrician should keep in mind threats of spontaneous abortion, preterm labour, cervical dystocia, etc.
\end{abstract}

Keywords: $3^{\circ}$ Uterine Prolapse, Morbidity

\section{INTRODUCTION}

Occurrence of prolapse of uterus during pregnancy is an extremely rare event. Less than 300 cases have been reported in the literature till date. Incidence of uterine prolapse in pregnancy is 1 in 10,000-15,000 deliveries worldwide. ${ }^{1}$ The hypothesis behind uterine prolapse is that uterine prolapse is precedent by elongation of cervix. $^{2}$ Although it is rare in occurrence but its importance lies in the fact that every obstetrician must know its management to ensure safe labour and delivery.

\section{CASE REPORT}

A 35 year old G5P3L1A1 with history of five months amenorrhea was referred to our hospital in view of uterine prolapse. On enquiring she gave history of something coming out of vagina since one month. Her obstetric history revealed that she had full term vaginal delivery in her first pregnancy. Intrapartum as well as immediate postpartum period was uneventful. After four months of delivery, her baby expired and the cause of death was unknown. In next pregnancy, she delivered a preterm female child, an IUD, vaginally at eight months of gestation. Following this, she had a normal vaginal delivery, baby was alive. She conceived again and had a spontaneous abortion at two months of gestation. Her past, personal and family history was insignificant. On examination, her vitals were stable. On per abdomen examination, fundal height corresponded to uterine height of 18 weeks, fetal parts were palpable and uterus was relaxed. On local examination, third degree uterovaginal prolapse with cord prolapse seen (Figure 1).There were no cord pulsations. Attendants were explained about the patient's condition. Patient went into labour spontaneously and expelled an abortus. She was 
discharged next day with advice regarding follow up visit after six weeks.

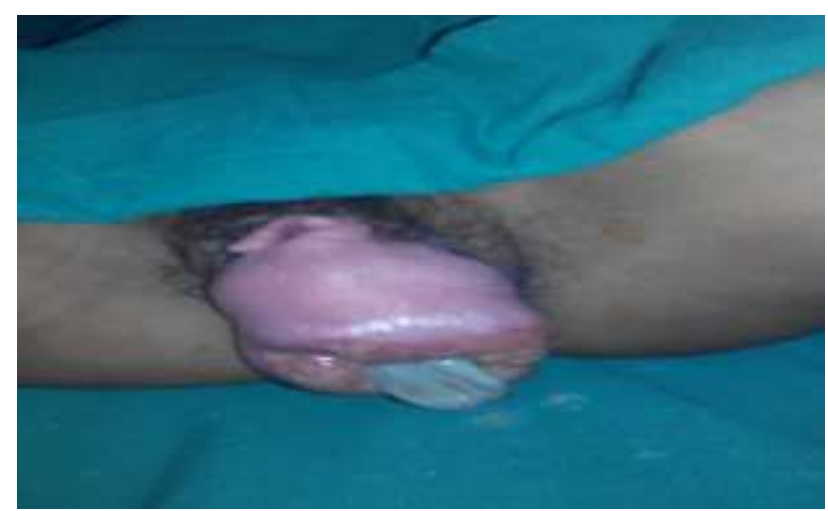

Figure 1: Third degree uterovaginal prolapse with cord prolapse.

\section{DISCUSSION}

Uterine prolapse during pregnancy is a rare occurrence which increases both maternal and fetal morbidity. There are many etiological factors which have been cited to be responsible for this condition, important ones are: Multiparity, vaginal delivery, advanced maternal age, increased body mass index. Other contributing factors are situations which lead to increased intra-abdominal pressure like straining, constipation, heavy weight lifting and chronic obstructive airway disease. ${ }^{3-8}$ Pre-existing prolapse is related to complications like infertility, spontaneous abortions, preterm labour. Due to venous obstruction and stasis, cervical edema occurs which may lead to mechanical trauma and cervical ulceration. Various studies report increased chances of urinary retention and thereby urinary tract infections in cases of pregnancy with uterine prolapsed. ${ }^{8-10}$ During labour there are more chances of cervical dystocia. Uterine rupture in lower segment also has been reported. ${ }^{11,12}$ Management of cases of pregnancy with uterine prolapse must be an individualized approach. Obstetrician must keep in mind above stated complications while deciding right approach for these patients. Bed rest with slight trendelenburg position is advisable for reposition of uterus and reduction of cervical edema. Perineal hygiene should be advised and in case of cervical ulcerations, local antibiotic application also can be recommended. After reduction of cervical edema, pessary is placed to keep the uterus in reduced position and shouldn't be removed until the onset of labour. ${ }^{8,10}$ Labour induction with misoprostol or oxytocin should be better avoided. Fundal pressure to expedite delivery should best be avoided otherwise it might lead to grave consequences. ${ }^{13,14}$ Elective caesarean section can be planned in cases with edematous and elongated cervix. $^{12,15,16}$ Individualized approach and careful monitoring is the key to manage such patients.

\section{CONCLUSION}

Uterine prolapse during pregnancy is a rare event. While managing such patients, an obstetrician should keep in mind threats of spontaneous abortion, preterm labour, cervical dystocia, etc. and warrants close observation and monitoring. However, Literature review shows consensus that conservative approach during pregnancy is the best way to deal with such cases and leads to a safe, spontaneous and uneventful delivery.

Funding: No funding sources

Conflict of interest: None declared

Ethical approval: Not required

\section{REFERENCES}

1. Yogev Y, Horowitz ER, Ben-Haroush A, Kaplan B. Uterine cervical elongation and prolapse during pregnancy: an old unsolved problem. Clin Exp Obstet Gynecol. 2003;30(4):183-5.

2. Partsinevelos GA, Mesogitis S, Papantoniou N, Antsaklis A. Uterine prolapse in pregnancy: a rare condition an obstetrician should be familiar with. Fetal Diagn Ther. 2008;24(3):296-8.

3. Van Dongen L. The anatomy of genital prolapse. S Afr Med J. 1981;60:357-9.

4. Jelovsek JE, Maher C, Barber MD. Pelvic organ prolapse. Lancet. 2007;369:1027-38.

5. Hendrix SL, Clark A, Nygaard I, Aragaki A, Barnabei V, McTiernan A. Pelvic organ prolapse in the Women's Health Initiative: gravity and gravidity. Am J Obstet Gynecol. 2002;186:1160-6.

6. Thakar R, Stanton S. Management of genital prolapse. BMJ. 2002;324:1258-62.

7. DeLancey JO. The hidden epidemic of pelvic floor dysfunction: achievable goals for improved prevention and treatment. Am J Obstet Gynecol. 2005;192:1488-95.

8. Brown HL. Cervical prolapse complicating pregnancy. J Natl Med Assoc. 1997;89:346-8.

9. Behringer FR, Vigilante M. Uterine prolapse at term. Obstet Gynecol. 1956;8:284-6.

10. Piver MS, Spezia J. Uterine prolapse during pregnancy. Obstet Gynecol. 1968;32:765-9.

11. Daskalakis G, Lymberopoulos E, Anastasakis E, Kalmantis K, Athanasaki A, Manoli A et al. Uterine prolapse complicating pregnancy. Arch Gynecol Obstet. 2007;276:391-2.

12. Pott-Grinstein E, Newcomer JR. Gynecologists' patterns of prescribing pessaries. J Reprod Med. 2001;46:205-8.

13. Merhi ZO, Awonuga AO. The role of uterine fundal pressure in the second stage of labour: a reappraisal. Obstet Gynecol Surv. 2005;60:599-603.

14. Tukur J, Omale AO, Abdullahi H, Datti Z. Uterine prolapse following fundal pressure in the first stage of labour: a case report. Ann Afr Med. 2007;6:194-6.

15. Partsinevelos GA, Mesogitis S, Papantoniou N, Antsaklis A. Uterine prolapse in pregnancy: a rare 
condition an obstetrician should be familiar with. Fetal Diagn Ther. 2008;24:296-8.

16. Sze EH, Sherard GB 3rd, Dolezal JM. Pregnancy, labour, delivery and pelvic organ prolapse. Obstet Gynecol. 2002;100:981-6.
Cite this article as: Latika, Nanda S, Chauhan M, Malhotra V. Pregnancy with third degree uterine prolapse-a rare case report. Int J Reprod Contracept Obstet Gynecol 2016;5:579-81. 